\title{
Femtosecond laser direct writing of three-dimensional micro/nanofluidics in porous glass
}

\author{
Ya Cheng ${ }^{1, *}$, Yang Liao ${ }^{1}$, and Koji Sugioka ${ }^{2}$ \\ ${ }^{I}$ State Key Laboratory of High Field Laser Physics, Shanghai Institute of Optics and Fine Mechanics, Chinese Academy of Sciences, \\ P.O. Box 800-211, Shanghai 201800, China \\ ${ }^{2}$ Laser Technology Laboratory, RIKEN - Advanced Science Institute, Hirosawa 2-1, Wako, Saitama 351-0198, Japan \\ Email: ya.cheng@siom.ac.cn
}

\begin{abstract}
In this contribution, we show that 3D micro-/nanofluidic components with arbitrary geometries and configurations can be directly formed inside glass by femtosecond laser direct writing. We further demonstrate that nanofluidic channels of a width of $\sim 40 \mathrm{~nm}$ can be directly fabricated in glass with linearly polarized femtosecond laser pulses, enabled by intentionally producing embedded nanograting structures with laser intensities slightly above a threshold value.
\end{abstract}

\section{Introduction}

Microfluidics provides unique capabilities of controlling and manipulating tiny volumes of liquids with high precision and ease of operation, thus it has enabled downsizing of both chemical and biological analysis [1, 2]. Femtosecond laser direct writing has been employed for creating 3D hollow structures embedded in glass which serves as microfluidic elements such as microchannels and microchambers. This 3D fabrication capability further opens up new avenues for fabricating a variety of integrated optofluidic microchips for chemical sensing and biological analysis. In this contribution, we focus on fabrication of micro-/nanofluidic structures with nearly arbitrary 3D geometries and configurations in porous glass [3-6].

\section{Fabrication of 3D microfluidics in porous glass}

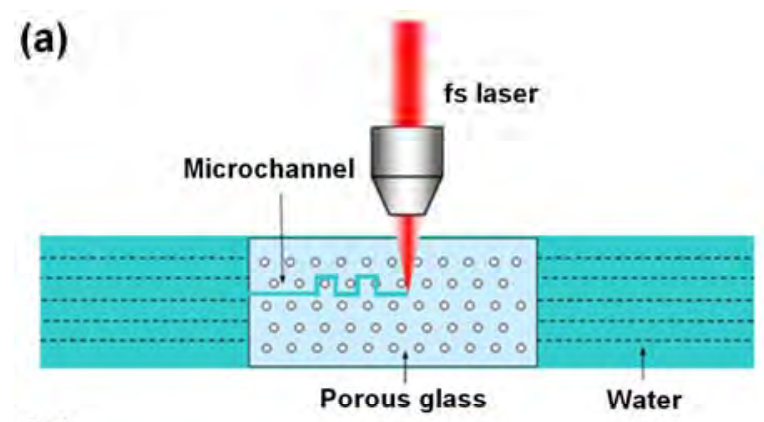

(b)

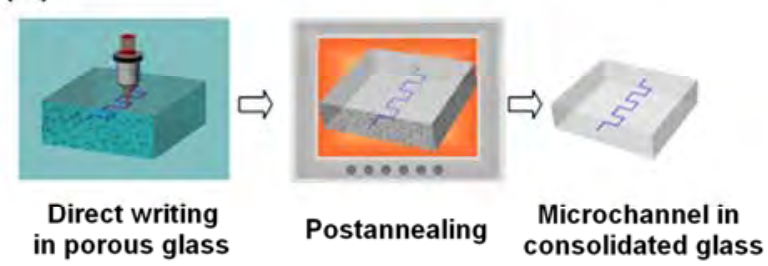

Fig. 1 (a) Schematic view of the experimental setup for water-assisted femtosecond laser direct writing inside porous glass, and (b) the flow diagram for the microchannel fabrication.

Fabrication of long 3D microfluidic channels with almost arbitrary lengths and configurations can be achieved by water-assisted femtosecond laser direct writing inside a porous glass. The porous glass substrates, which have a size of $15 \times 15 \times 3 \mathrm{~mm}$, were produced by removing the borate phase from phase-separated alkali-borosilicate glass in hot acid solution. The schematic of the water-assisted femtosecond laser direct writing inside porous glass is 
illustrated in Fig. 1(a). The porous glass was immersed in distilled water. The femtosecond laser beam was tightly focused into the glass sample for inducing internal ablation. During the ablation, bubbles were continuously produced around the focal point which contain the laser-ablation-generated debris. When the bubbles were driven out from the openings at the surface of the glass sample, the debris in the microfluidic channel could be efficiently removed. 3D microchannels were fabricated by slowly translating the glass sample according to preprogrammed patterns [3-5]. After the laser direct writing, the sample was placed in a furnace and annealed at a temperature of $1150{ }^{\circ} \mathrm{C}$ to consolidate into a compact glass, as shown in Fig. 1(b).

\section{Fabrication of nanofluidics}

Very recently, nanofluidic channels have also been created inside porous glass by femtosecond laser direct writing, as shown in Figs. 2(a) and 2(b). Control of the diameter of the nanochannel is achieved by adjusting the pulse energy of the writing beam [6]. Here, we achieve fabrication resolutions far beyond diffraction limit (i. e., 40 nm) by combining the threshold effect and the exotic phenomenon of formation of periodic nanograting during the process of laser writing in the porous glass immersed in water. In the porous glass, we observe that the nanogratinglike structures induced by femtosecond laser irradiation constitute an array of hollow cracks with a width down to $\sim 40 \mathrm{~nm}$ [6]. When the femtosecond laser intensity is intentionally reduced to a level at which only the hollow nanocrack produced in the central area of the focal volume can survive, a single-line nanofluidic channel is produced, as shown in Fig. 2(b).

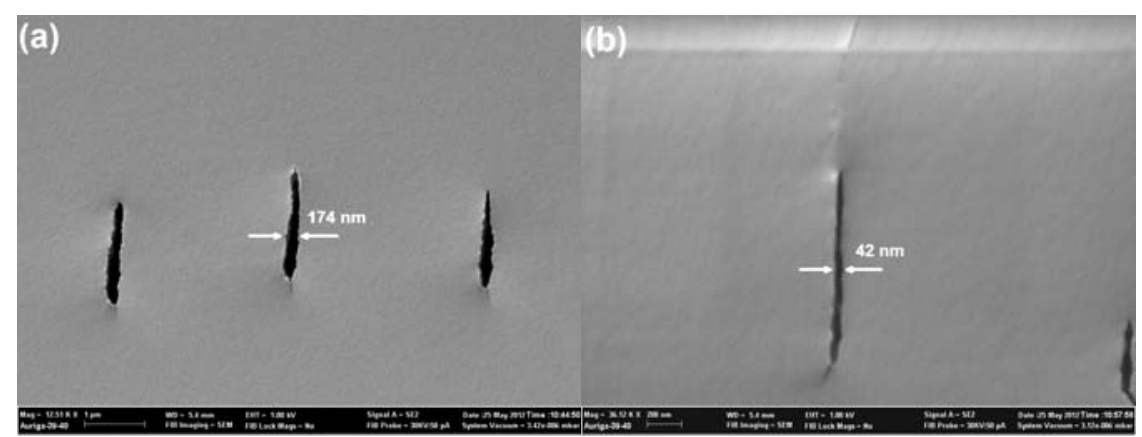

Fig.2 Nanofluidic channels written in porous glass immersed in water using a $40 \mathrm{fs}, 800 \mathrm{~nm}, 250 \mathrm{kHz}$ femtosecond laser at different pulse energies. (a) $140 \mathrm{~nJ} /$ pulse; (b) $128 \mathrm{~nJ} /$ pulse. Writing speed: $10 \mu \mathrm{m} / \mathrm{s}$; objective lens: water-immersed objective (N.A.= 1.10). Substrates have been postannealed for collapsing all the nanopores in the glass.

\section{Conclusions}

In conclusion, we have demonstrated the fabrication of 3D micro-/nanofluidics in porous glass with extreme flexibilities in terms of channel dimensions and geometries. In particular, the 3D nanofluidic channels fabricated in glass may open many opportunities in a variety of fields of research, such as nanofluidic physics and chemistry, DNA analysis, lab on a chip, nanoplasmonics, and so forth.

\section{References}

[1] A. Manz, N. Graber and H.M. Widmer (1990), Miniaturized total chemical analysis systems: A novel concept for chemical sensing, Sensor. Actuat. B, 1, 244-248.

[2] G.M. Whitesides (2006), The origins and the future of microfluidics, Nature, 442, 368-373.

[3] Y. Liao, Y. Ju, L. Zhang, F. He, Q. Zhang, Y. Shen, D. Chen, Y. Cheng, Z. Xu, K. Sugioka and K. Midorikawa (2010), Three-dimensional microfluidic channel with arbitrary length and configuration fabricated inside glass by femtosecond laser direct writing, Opt. Lett., 35, 3225-3227.

[4] Y. Ju, Y. Liao, L. Zhang, Y. Sheng, Q. Zhang, D. Chen, Y. Cheng, Z. Xu, K. Sugioka and K. Midorikawa (2011), Fabrication of large-volume microfluidic chamber embedded in glass using three-dimensional femtosecond laser micromachining, Microfluid. Nanofluid., 11, 111-117.

[5] C. Liu, Y. Liao, F. He, Y. Shen, D. Chen, Y. Cheng, Z. Xu, K. Sugioka and K. Midorikawa (2012), Fabrication of three-dimensional microfluidic channels inside glass using nanosecond laser direct writing, Opt. Express, 20, 4291-4296.

[6] Y. Liao, Y. Shen, L. Qiao, D. Chen, Y. Cheng, K. Sugioka and K. Midorikawa, Femtosecond laser nanostructuring in glass with sub-50nm feature sizes, submitted 